early developmental stages, for a complete definition of cell types.

How identity progresses with time in postmitotic neurons remains elusive. It will be particularly useful to dissect the extent to which external factors, including sensory signals from the peripheral nervous system, drive differentiation and further diversification of neurons within these genetically poised subtypes. Resolving these issues will be central not only to addressing the mechanisms that generate neuronal diversity, but also to characterizing and understanding inter-individual differences in circuit structure and function.

Finally, both studies identify disease-related genes among the cell-type-specific transcripts, including some associated with autism and schizophrenia. As such, they provide a valuable resource for those seeking to understand the mechanisms underlying these disorders.

Ludovic Telley is in the Department of Fundamental Neuroscience, University of Lausanne, 1005 Lausanne, Switzerland. Denis Jabaudon is in the Department of Basic Neurosciences, University of Geneva, 1211 Geneva, Switzerland. e-mails:ludovic.telley@unil.ch; denis.jabaudon@unige.ch
1. Mayer, C. et al. Nature 555, 457-462 (2018).

2. Nowakowski, T. J. et al. Science 358, 1318-1323 (2017).

3. Jabaudon, D. Nature Commun. 8, 16042 (2017).

4. Rakic, P. Science 241, 170-176 (1988)

5. O'Leary, D. D. M. Trends Neurosci. 12, 400-406 (1989).

6. Puelles, L. \& Rubenstein, J. L. R. Trends Neurosci. 26, 469-476 (2003).

7. Johnson, M. B.\& Walsh, C. A. Curr. Opin. Neurobiol. 42, 9-16 (2017).

8. Wamsley, B. \& Fishell, G. Nature Rev. Neurosci. 18, 299-309 (2017).

9. Radick, G. Nature 533, 293 (2016).

10.Weldon, W. F. R. Biometrika 2, 44-55 (1902).

11.Mi, D. et al. Science http://dx.doi.org/10.1126/ science.aar6821 (2018)

This article was published online on 5 March 2018.

\title{
A cellular passage to the root interior
}

Water-conducting tissues inside plant roots are surrounded by impermeable cells. This protective barrier is punctured by 'passage cells', which are thought to regulate nutrient uptake. How these cells form has now been revealed. SEE LETTER P.529

\section{SEDEER EL-SHOWK \& ARI PEKKA MÄHÖNEN}

$\mathrm{P}$ lants need to take up water and nutrients through their roots, while keeping out pathogens and toxins. To achieve this, the roots' inner transport tissues are enclosed in a protective, impermeable barrier of endodermal cells, interspersed with 'openings' in the form of a specific type of endodermal cell called a passage cell, which is thought to serve as a cellular gatekeeper, controlling access to the root interior ${ }^{1-4}$. On page 529, Andersen et al. ${ }^{5}$ describe molecular mechanisms that control the formation of passage cells, and show how the numbers of such cells are regulated by nutrient availability. The findings offer insights into the formation of these key plant cells, and link this to the patterning processes that form the embryonic root, providing an intriguing demonstration of the continuity of developmental mechanisms.

As endodermal cells develop, the impermeable polymers lignin and suberin are deposited in the cell walls. The deposits build up as the cells mature, forming an impermeable barrier ${ }^{4}$ that isolates the adjacent inner tissues from the root's outer cell layers and the soil. Once this process is complete, it is thought that nutrients and water can reach the interior only by means of passage cells ${ }^{4}$. These cells contain lignin deposited in an arrangement called the Casparian strip in their cell walls, but lack suberin, and thus offer a permeable route for molecular transport ${ }^{4,5}$. The extent of 'suberization' of endodermal cells is regulated by the

levels of the hormones abscisic acid (ABA) and ethylene, and can be reversibly affected by both nutrient availability and stress ${ }^{6}$. The extent of suberization is thought to be a key factor in the rate of nutrient uptake ${ }^{6}$. Although the role and regulation of suberization in endodermal cells are understood, the molecular mechanisms controlling the development of passage cells have remained a mystery.

Passage cells have been observed in many plants species ${ }^{1-3}$, and are formed in a region of the root above the growing root tip. Andersen et al. used the plant

"Plants can dynamically adjust the number of passage cells according to nutrient status." Arabidopsis thaliana as their model system. To confirm that passage cells form in its roots, they tracked the expression of a gene required for suberin synthesis by monitoring a fluorescent marker protein. They identified suberindeficient passage cells distributed at seemingly random locations along the root's length. The cells were consistently positioned around the root's circumference near the developing xylem, one of two types of water- and nutrient-conducting tissue (Fig. 1).

To investigate the nature of the link between xylem and passage cells, the authors tested plants in which genetic mutations altered the pattern of xylem development. Two of the mutant plants ${ }^{7,8}$ had fewer passage cells than normal. These plants had defects in the genes
AHP6 or LOG4, which encode proteins that act respectively in the signalling and biosynthesis of the hormone cytokinin. Cytokinin affects the transport and signalling of the hormone auxin to regulate the development and patterning of water-conducting tissues ${ }^{8,9}$. Auxin, in turn, drives the expression of AHP6, which encodes a protein that inhibits cytokinin signalling in the developing xylem ${ }^{7,9}$. The authors found that AHP6 protein (fused to a fluorescent marker protein) diffused from the developing xylem into the adjacent endodermal cells, probably through cell-connecting nanostructures called plasmodesmata, and that this process was required for passage-cell formation.

The finding led Andersen and colleagues to suspect that a feedback loop between cytokinin and auxin signalling might be involved in passage-cell formation. They therefore analysed plants expressing marker proteins to monitor the levels of cytokinin signalling. They found that passage cells have low levels of cytokinin signalling, or lack it altogether, unlike their neighbouring suberized endodermal cells. Analysis of another marker protein showing the level of auxin signalling revealed an auxin response in all of the endodermal cells near the developing xylem, including passage cells. Seedlings grown with auxin had more passage cells than did plants that did not receive an auxin boost. Conversely, seedlings treated with cytokinin contained fewer passage cells than untreated seedlings. Opposing roles for auxin and cytokinin are a ubiquitous theme in plant development, and the presence of such a feedback loop links passage-cell formation to patterning processes acting in the transport tissues and elsewhere.

The authors investigated whether auxin and cytokinin might affect ABA-mediated suberin deposition. Plants that were engineered to express cytokinin-signalling inhibitors throughout the endodermis had low suberin deposition in all endodermal cells, and this outcome was unaffected by the addition of ABA. However, it is unclear why ABA-mediated suberin deposition requires cytokinin signalling. Plants were engineered to express auxin-signalling inhibitors throughout the 


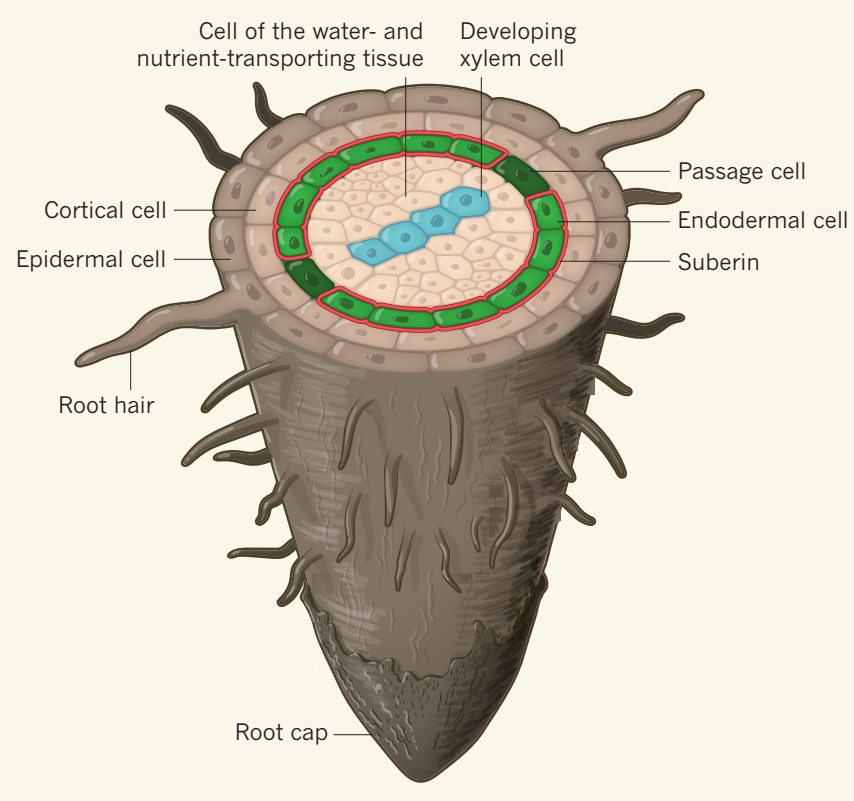

Figure 1 | Passage cells in the plant root. A protective layer of endodermal cells, rendered impermeable by deposition of the polymer suberin (red), surrounds the water- and nutrient-transport tissues at the core of the plant root. But certain endodermal cells, known as passage cells, do not contain suberin, and these cells have been proposed as cellular gatekeepers that control how water and nutrients reach the inner root tissues ${ }^{1-4}$. Andersen et al. ${ }^{5}$ report studies in the model plant Arabidopsis thaliana that shed light on how passage cells develop. The authors find that the cells form adjacent to the region of developing xylem (blue), which is part of the plant's transport tissues. The protein AHP6 (not shown) moves from the developing xylem into endodermal cells. It inhibits the signalling pathway mediated by the hormone cytokinin, and prevents suberin from being deposited in the cell walls in response to the hormone abscisic acid (not shown). The lack of suberin enables the formation of passage cells.

endodermis, the number of passage cells was reduced compared with controls, and ABA addition had no effect on this, either.

Andersen and colleagues propose that most endodermal cells usually have a high level of cytokinin signalling activity, enabling them to undergo ABA-mediated suberization, and that these cells eventually reach a level of suberization that makes them impermeable. However, endodermal cells adjacent to the developing xylem receive AHP6, resulting in low levels of cytokinin signalling, and these cells also have high auxin signalling. These characteristics render some of the cells unresponsive to $\mathrm{ABA}$, so they remain unsuberized and form passage cells. The authors suggest that stochastic differences in cytokinin-mediated ABA sensitivity may determine which of the cells become passage cells, but it will be fascinating to discover whether this process is fine-tuned by other factors, such as nutrient sensing.

Additional work will be required to elucidate the genetic and hormonal interactions that mediate this patterning network. Does cytokinin regulate sensitivity to $\mathrm{ABA}$ in the endodermis, and do auxin transporter proteins concentrate auxin to aid passage-cell formation? Alternatively, is auxin distribution in the endodermis determined by the auxin dynamics in the underlying transport tissues ${ }^{10}$ ?
Andersen and colleagues also investigated passage-cell function. Although it has been proposed that these cells are involved in nutrient intake, only a few studies have investigated their role $e^{4,5}$. A previous study ${ }^{11}$ in $A$. thaliana roots found that the expression of a gene encoding a phosphate transporter protein is correlated with the location of passage cells. Andersen and colleagues confirmed this finding, and showed that the transporter gene and several related genes are expressed in adjacent cortical and epidermal cells in the outer layers of the root. This pattern of phosphate transporters suggests that phosphate, and perhaps other nutrients, might be funnelled through passage cells towards the water- and nutrienttransport system.

The authors found that nutrient deficiency leads to more-widespread expression of the phosphate transporter in xylem-associated endodermis and a reduction in suberization of these cells. This shows that plants can dynamically adjust the number of passage cells according to a plant's nutrient status. Finally, Andersen et al. discovered that monitoring the expression of phosphate transporters allowed passage cells to be identified at an early stage of development. Identification occurred before the initial deposition of suberin in neighbouring endodermal cells that do not form passage cells, providing a tool for future studies of the early events in passage-cell formation.

This latest work opens the door to further investigation of the role of passage cells. Genetic manipulation of passage-cell formation using the mutants and genetic tools developed in this study could be used to investigate the spectrum of nutrients that might be transported through passage cells, as well as to assess the cells' role in nutrient uptake. Another area for future research would be to investigate whether these cells are a potential entry point for microorganisms that cause disease, or those that form a mutually beneficial relationship with plants ${ }^{2}$.

It is interesting that the key genes modulating the cytokinin and auxin fluxes involved in passage-cell formation also act in the patterning of the embryonic root ${ }^{8}$ and waterconducting tissues?. Plant development is a continuous process that probably requires developing structures to integrate information from hormonal signalling pathways acting at different times and in different parts of the plant. A key question is how the dynamics of the hormonal signalling interactions that drive so many aspects of plant development are integrated to link these developmental processes.

Sedeer el-Showk is a freelance science writer based in Ain Aouda, Morocco. Ari Pekka Mähönen is at the Institute of Biotechnology, HiLIFE, University of Helsinki, Helsinki 00014, Finland, and in the Faculty of Biological and Environmental Sciences, University of Helsinki.

e-mails: sedeer@elshowk.com; aripekka.mahonen@helsinki.fi

1. Kroemer, H. Wurzelhaut: Hypodermis und Endodermis der Angiospermenwurzel (Nägele, 1903).

2. Peterson, C. A. \& Enstone, D. E. Physiol. Plant. 97, 592-598 (1996).

3. Wu, H., Jaeger, M., Wang, M., Li, B. \& Zhang, B. G. Ann. Bot. 107, 843-853 (2011).

4. Robbins, N. E. II, Trontin, C., Duan, L. \& Dinneny, J. R. Plant Physiol. 166, 551-559 (2014).

5. Andersen, T. G. et al. Nature 555, 529-533 (2018).

6. Barberon, M. et al. Cell 164, 447-459 (2016).

7. Mähönen A. P. et al. Science 311, 94-98 (2006).

8. De Rybel, B. et al. Science 345, 1255215 (2014).

9. Bishopp, A. et al. Curr. Biol. 21, 917-926 (2011).

10.el-Showk, S. et al. PLoS Comput. Biol. 10, e1004450 (2015).

11. Hamburger, D., Rezzonico, E., MacDonald-Comber Petétot, J., Somerville, C. \& Poirier, Y. Plant Cell 14, 889-902 (2002).

This article was published online on 14 March 2018.

\section{CORRECTION}

The News \& Views 'A surprising chill before the cosmic dawn' by Lincoln Greenhill (Nature 555, 38-39; 2018) incorrectly said in the seventh paragraph that a putative absorption signal looks back as far as 180 million years ago. This should have said "as far back as 180 million years after the Big Bang". 